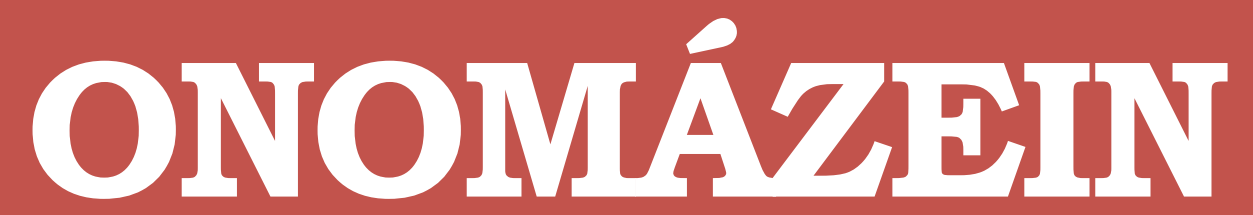

Journal of linguistics, philology and translation
PONTIFICIA UNIVERSIDAD CATÓLICA DE CHILE FACULTAD DE LETRAS

\title{
Digital editing of Early Modern English medical manuscripts: scribal errors and corrections
}

\author{
Laura Esteban-Segura \\ Universidad de Málaga \\ España
}




\section{Abstract}

An important philological question is how to edit texts. An edition always entails interpretation of the text and also of the sociocultural context in which the manuscript was created and used. In new philological theory, and contrary to more traditional approaches, the individual manuscript versions, i.e., the textual witnesses, are regarded as valuable in their own right, as every textual witness tells us something about the culture of manuscripts (Carlquist, 2004: 112). This is the approach followed for the digital editing of Early Modern English scientific writing in The Malaga Corpus of Early Modern English Scientific Prose. In this paper, we discuss the challenges that producing such type of edition pose. We will particularly focus on the issue of scribal errors and corrections and how the editor can treat and capture them in the edition. MS Wellcome 213, one of the texts included in the above-mentioned corpus, will be analysed for the purpose. The corpus consists of manuscripts from the Hunterian Collection (Glasgow University Library), the Wellcome Collection (London Wellcome Library) and the Rylands Collection (University of Manchester Library). With regard to text types, these manuscripts hold specialized texts, surgical and anatomical treatises, as well as recipe collections and materia medica.

Keywords: scribal errors and corrections; digital editing; Early Modern English; MS Wellcome 213. 


\section{Introduction}

This paper examines the occurrence of scribal mistakes and the different methods employed to correct them in London, Wellcome Library, MS Wellcome 213. ${ }^{1}$ The manuscript, written in Early Modern English and dating from the early 17th century (1606, more specifically), contains medical recipes. We will also discuss how the mistakes and corrections can be handled in a digital edition.

The text has already been transcribed and its edition is being currently prepared as part of a research project, entitled The Malaga Corpus of Early Modern English Scientific Prose (CalleMartín and others, 2017), ${ }^{2}$ which aims at the electronic editing of so far unedited Early Modern English scientific manuscripts housed in the Hunterian Collection at Glasgow University Library, the Wellcome Collection at the Wellcome Library in London and the Rylands Collection at the University of Manchester Library. Both the digitised images and the corresponding semidiplomatic transcriptions are provided. A second objective of the project is to compile a POS-tagged corpus of Early Modern English Fachprosa.

Four main stages can be distinguished in the development of the project: first, the manuscripts were selected and digitised; then, the semidiplomatic transcriptions and physical descriptions of the treatises under consideration were carried out; linguistic analysis and corpus annotation conform the third stage; and finally, the last stage involves the electronic publication of the editions.

The interest in scientific material has both linguistic and extralinguistic motivations. On linguistic grounds, scientific prose in the vernacular is considered to be less artificial than poetry or drama, and thus it is an appropriate input for linguistic analysis. Extralinguistically, on the other hand, most of this material is unedited and therefore the access to it is limited, so it can be made available to the scientific community and/or society in general.

The evolution of science during Early Modern English implied a reorganisation of the medical writing in the period that, helped by book production, spread widely among the society of the period, especially after 1550 (Taavitsainen and others, 2011: 9-11). This not only triggered the expansion of already existent text types, but also the incorporation of new ones to those already present in Late Middle English.

1 Research for this paper has received financial support from the Spanish Ministry of Economy and Competitiveness (grant number FFI2014-57963-P) and from the Autonomous Government of AndaIusia (grant number P11-HUM-7597). I am grateful to the Wellcome Library for kindly permitting the reproduction of images from the manuscript (their copyright resides with the Wellcome Library).

2 The project can be consulted at https://modernmss.uma.es/. 
Taavitsainen and others (2011: 22-25) provide a classification of Early Modern medical writing into: (i) general treatises or textbooks, which offer a systematic account of the whole field of medicine; (ii) treatises on specific topics, such as diseases, methods of diagnosis or treatment, therapeutic substances, midwifery and children's diseases, and the plague; (iii) surgical and anatomical treatises; (iv) recipe collections and materia medica, which contain both remedy books and formalised pharmacopoeias; (v) regimens and health guides, made up of texts on preventive medicine; and (vi) Philosophical Transactions, which consist entirely of medical texts published as letters or articles in the Philosophical Transactions of the Royal Society. This text type is new to the Early Modern English period, whereas the first two correspond to the specialised texts of the Late Middle English classification.

In spite of the advent of writing, there were certain text types that were more likely to be handwritten, such as the tailored compilation of remedies from various sources, students' notebooks and records of the different treatments that a particular physician applied to his patients (Taavitsainen and others, 2011: 10-11).

As mentioned previously, The Malaga Corpus of Early Modern English Scientific Prose includes manuscripts from the Hunterian Collection (Glasgow University Library), the Wellcome Collection (Wellcome Library, London) and the Rylands Collection (University of Manchester Library). They hold specialized texts, both general and specific treatises (e.g., on the diseases of women or on the anatomy of the eye), surgical and anatomical texts, and recipe collections, thus affording a comprehensive view of Early Modern English medical prose.

On the website, which is quite intuitive, the digitised manuscripts can be freely consulted along with their semidiplomatic transcriptions, which can be useful for those not fully acquainted with the basics of historical palaeography. User-friendly tools allow: a) to magnify the images; b) to search for the occurrences of any word; and c) to check the transcribed text against the transcribed image. Figure 1 shows a page from MS Wellcome 213 together with its transcription.

When it comes to editing texts, there are questions that the editors should ask themselves because an edition involves interpretation of both the text and of the "social culture in which the manuscript was produced and consumed” (Carlquist, 2004: 112).

In traditional editions, the editor takes into consideration extant versions of a text and tries to interpret what the text was meant to be, hence producing a hypothetical version of it. By comparing the extant copies the editor prepares a textual stemma. This method, textual criticism, goes back to the nineteenth century and works especially for literary texts. The abstract text is in focus and the concrete versions are less important. In new philological theory, however, the individual manuscript versions, the textual witnesses, are regarded as valuable in their own right, as every textual witness tells us something about the culture of manuscripts (2004: 112). This is the approach that we have followed in our project. Thus, we have retained 


\section{FIGURE 1}

Example of digitised manuscript (MS Wellcome 213, p. 3)

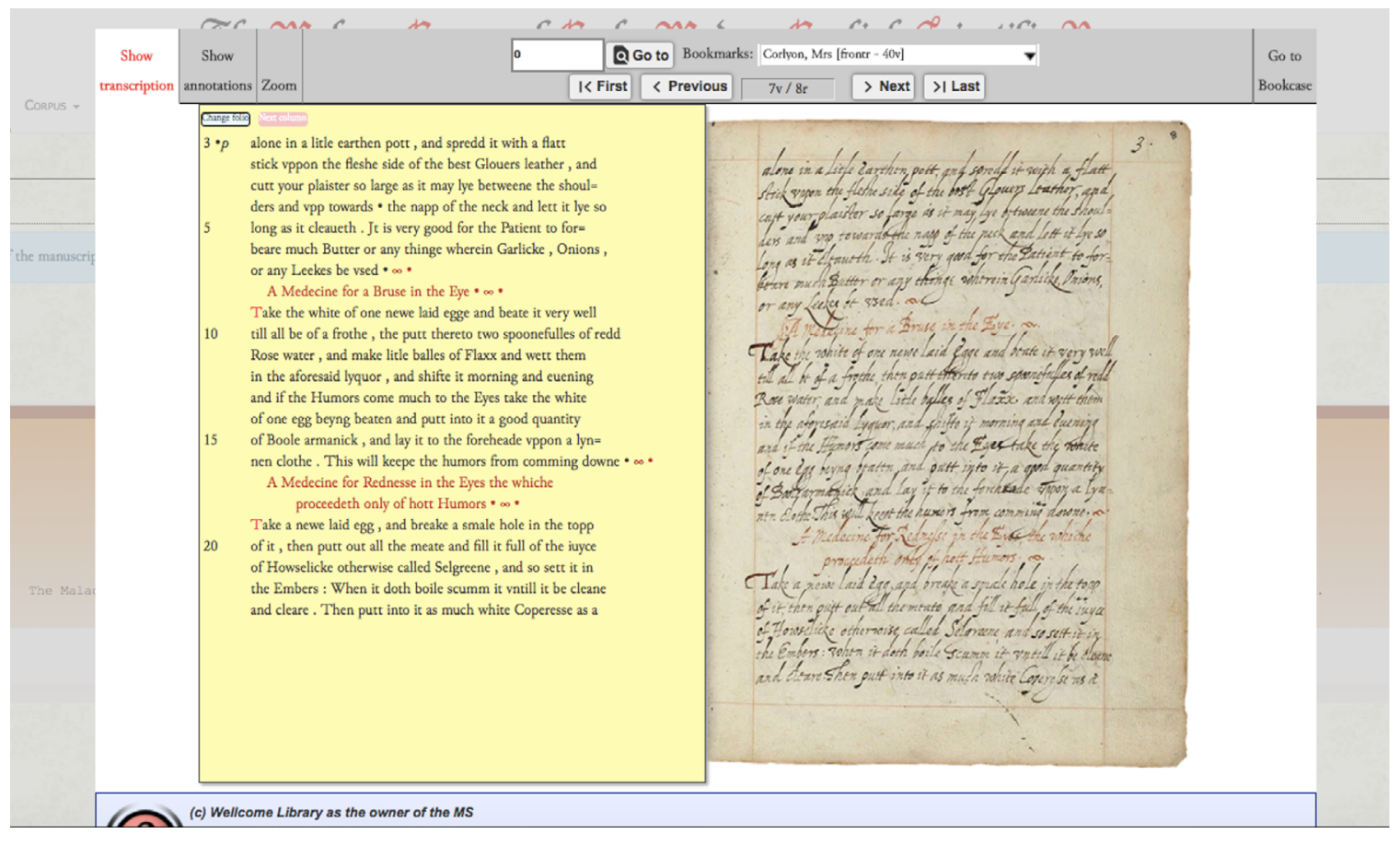

original spelling although normalisation is a common editorial practice. We have tried to explicitly indicate editorial intervention at all times by using italics to mark it, for example, the expansion of abbreviations. In this fashion, editorial interventions in the text are visible and the original form is recoverable. The original punctuation as well as capitalisation and word division have been kept. Nevertheless, we have marked every five lines in the margins for ease of consultation and the number of page in the case of front pages appears on the left instead of on the right.

\section{The manuscript}

MS Wellcome 213 is a recipe book which comprises diverse medicines, broths, salves, waters, syrups and ointments of which many or the most part have been experienced and tried by the special practice of Mrs Corlyon, who is the compiler, as stated in the first page ("A Booke of diuers Medecines, Broothes, Salues, I Waters, Syroppes and Oyntementes of which many or | the most part haue been experienced and tryed | by the speciall practize of | $M^{\text {rs }}$ Corlyon"; see figure 2). This first page of the manuscript (taking into account the new pagination; see below) is in fact very informative not only concerning contents but also ownership. Above the title, and in the same hand, is written "Liber Comitissæ Arundeliæ" ("Book of the Countess of Arundel”; see figure 2). This refers to Alethea Talbot, Countess of Arundel and Surrey, wife of Thomas Howard, second 
Earl of Arundel. The volume, beautifully inscribed and gilt-stamped with the 'AA' monogram of Alethea Arundel, is believed to have been a wedding gift from her mother-in-law, Anne Dacre, on the occasion of Alethea's marriage to his son, Thomas Howard, in 1606 (Leong and Pennell, 2007: 141). The Countess was a collector of medical and culinary recipes (Rabe, 2016: 187) and her collection of recipes, probably based on the contents of Wellcome 213, was published as a book entitled Nature Exenterata: or Nature Embowelled... in London in 1655.

\section{FIGURE 2}

Contents and ownership (MS Wellcome 213, p. 1)

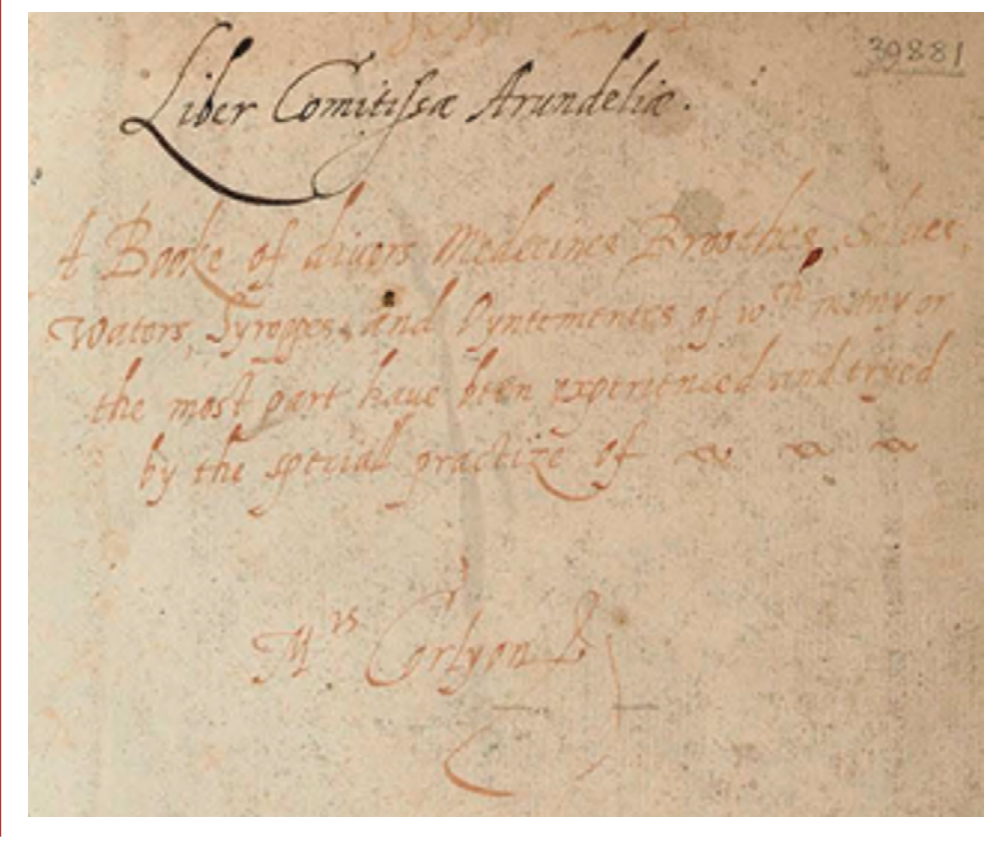

The manuscript has been paginated twice. The old pagination is contemporary to the text and includes 350 pages; some pages have deliberately been left blank. The pages have been renumbered in pencil and this new pagination consists of 194 pages. Before the text, there is a one-page table of contents making reference to all the chapters or titles. A more detailed table of contents (15 pages long) including all the particular medicines and parts of the body is found at the end of the manuscript. For the text, black ink is employed, whereas heading titles and letters at the beginning of paragraphs are in red ink. This suggests a meticulous process of copying. Although the handwriting of the text is good and careful, it presents some mistakes and, in the majority of cases, corrections to them.

\section{Scribal errors}

It is at times difficult to determine what exactly made up an error in vernacular works in the Late Medieval and Early Modern period because the system of norms on spelling and 
punctuation was not as clear-cut as in Present-Day English, and syntax and grammar were not strict. We find, for instance, different spellings for the same word in the manuscript, such as both "vpon" and "vppon" for "upon". Therefore, even when an author's stylistic habits are fairly predictable, it cannot be presumed that a different tense, a more common spelling or the inclusion of a particular mark of punctuation were intended or altered by the scribe (Petti, 1977: 29).

Moorman (1975: 57) classifies errors into two categories: spontaneous or unconscious variation, such as a simple slip of the pen, on the one hand, and determined or conscious variation, on the other, which is generally an attempt at emendation (correct or not) of the copy being transcribed. Most errors belong to the first category. In the following subsections we will deal with the types of errors found in Wellcome 213.

\subsection{Omission}

One of the most common forms of error is omission, which can occur as a consequence of the scribes confusing their points of reference. The presence of similar words or phrases, optical illusion, etc., can also account for omission. As the very name suggests, it entails the loss of letters, words and even phrases. An instance from the manuscript is offered in figure 3 , in which the noun "redd" has been omitted (and later corrected): "before your meate, drinck smale drinck and alwayes put a gadd | of steele $\wedge$ <redd $\rangle$ hott into the same. At after noones lay you downe to" (p. 144; emphasis added) ("before your meal, drink small drinks and always put a goad of red hot steel into the same. In the afternoon, lay down to...").

\section{FIGURE 3}

Example of omission

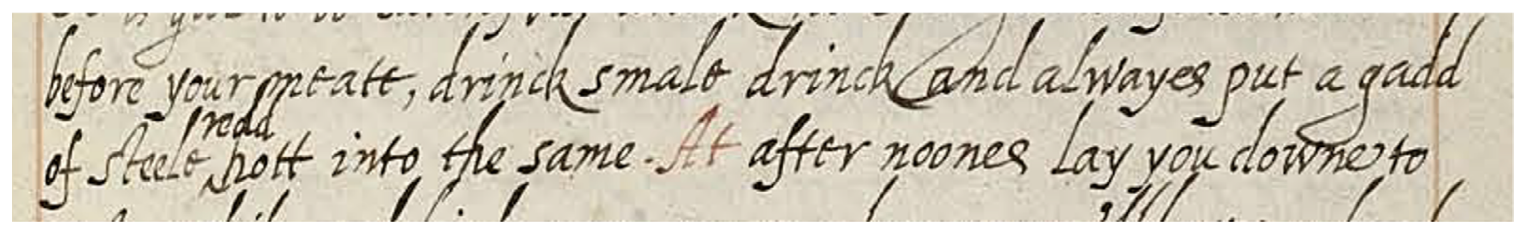

\subsection{Addition}

Additions, which are syllables, words or phrases written twice, represent another type of error. Lapse of memory or visual fatigue can explain this mechanical repetition, which frequently goes unnoticed by the scribe since no correction is undertaken.

An example of addition from the manuscript is the repetition of the article "the", as shown in figure 4. Here the mistake can be justified by the ending of line: "by this: Jt dymmeth the sighte, at the beginninge the | the eye looking very fayre: The black of the Eye soone" (p. 8; 
emphasis added) ("by this: it dims the sight, at the beginning the *the eye looking very fair: the black of the eye soon...”).

\section{FIGURE 4}

Example of addition

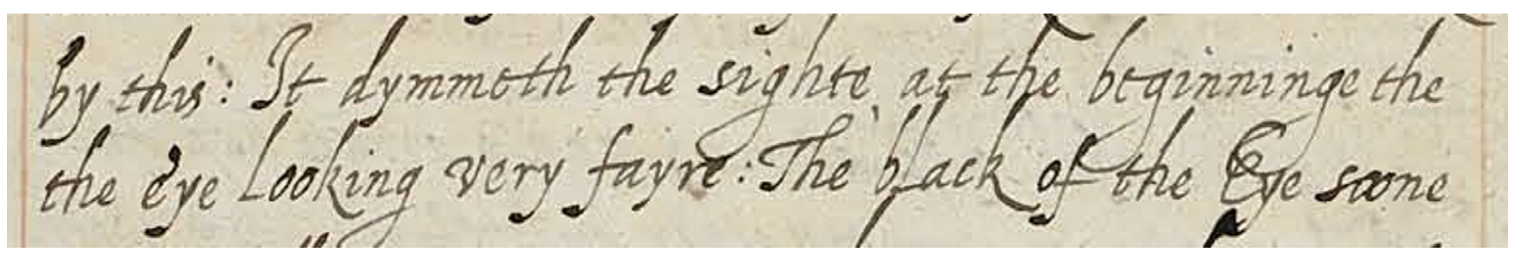

\section{Scribal corrections}

Once the types of errors encountered in the manuscript have been examined, the methods of correction made use of by the scribe during the process of copying the text will be discussed. Unfortunately, in the Early Modern English period there was not a delete key, tippex or a very effective eraser to remove ink. Therefore, pages with mistakes on them were not discarded and corrections were undertaken. The manuscript exhibits two methods of correction: deletion, which includes cancellation and obliteration, and insertion.

\subsection{Deletion}

\subsubsection{Cancellation}

There are several types of deletion. One of them is cancellation, which usually takes the form of one or two straight lines, as illustrated in figure 5: "Take a greate handfull of Peache flowers, of wilde Dasye I rootes and of the whitest * of wilde Tansye of ech of these the" (p. 42; emphasis added) ("Take a great handful of peach flowers, of wild daisy roots and of the whitest *of wild tansy, of each of these the...").

\section{FIGURE 5}

Example of cancellation (i)

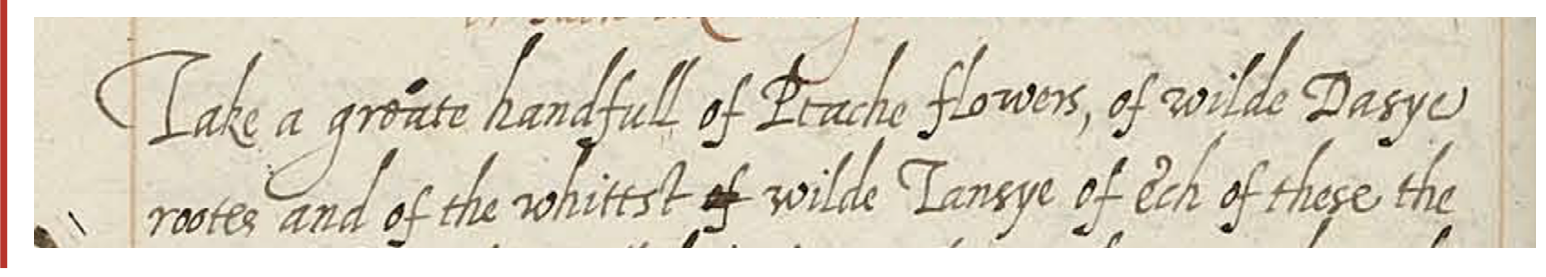

Cancellation can also involve crosses through the centre of the words or letters to be deleted roughly parallel to the base-line. If there is a correction to be made, this can appear written 
next. An example of cancellation with crosses is offered in figure 6 (in the word "handful"): "good quantity of bay Salte and of the Oyle of Dyll and of the | Oyle of Hearbegrase of eche of these a good *handfull spoonefull" (p. 139; emphasis added) ("good quantity of bay salt and of the oil of dill and of the oil of herbage, of each of these a good *handful spoonful").

\section{FIGURE 6}

Example of cancellation (ii)

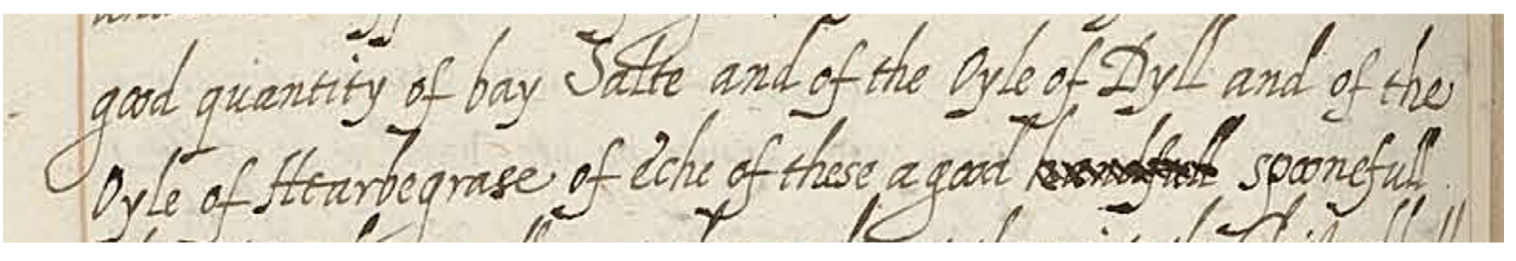

\subsubsection{Obliteration}

In obliteration, the error is covered with ink either by blotting, blurring or obscuring the letter or word. The correction generally appears next to it or above, as shown in figures 7 ("vntill almost the one halfe be consumed, then take $\wedge\langle i$ it of *it" [p. 76; emphasis added] > "until almost the one half is consumed, then take $\wedge\langle$ it $>$ of *it") and 8 ("Patient drinck of this powder oftentimes $\wedge\langle$ or $\rangle$ *of of $\mathrm{S}^{\mathrm{t}}$ Johns woorte" [p. 154; emphasis added] > "Patient drink of this powder often $\wedge\langle 0 r\rangle{ }^{*}$ of of St John's wort"). Obliteration has been described as unsophisticated, rarely found in formal manuscripts, but common in rough drafts (Petti, 1977: 29).

\section{FIGURE 7}

Example of obliteration (i)

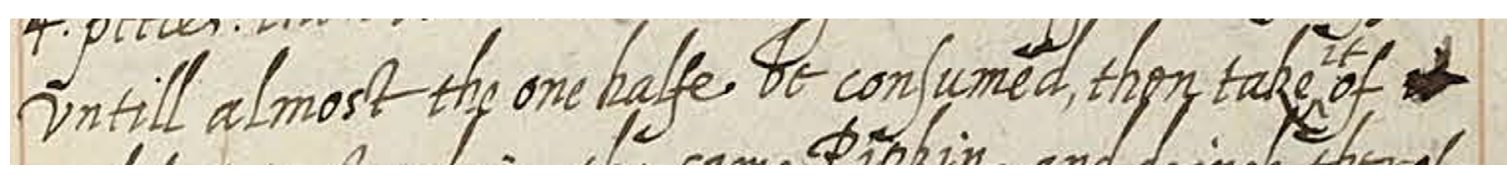

\section{FIGURE 8}

Example of obliteration (ii)

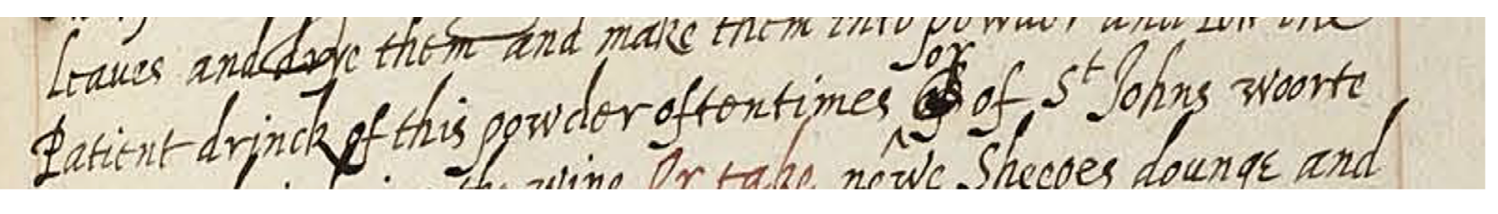

The margins can also be used to write the correction when there is not enough space in the text. In figure 9, the scribe has mistakenly written the abbreviation of "dram", a unit of apoth- 
ecary weight, whereas the correct abbreviation should have been the one corresponding to the unit "ounce", whose symbol appears in the left margin ("a Plaister, then putt vnto it of Emplastrum Dia=| chilon cum gummis *dram ij of Olei Jrini Linacei of ech | two ounzes Of Cummyn seede in fyne Powder fower" [p. 317; emphasis added] > "a plaster, then put into it: of emplastrum diachilon cum gummis, two *drams; of olei jrini linacei of each, two ounces; of cumin seed in fine powder, four...").

\section{FIGURE 9}

Example of obliteration (iii)

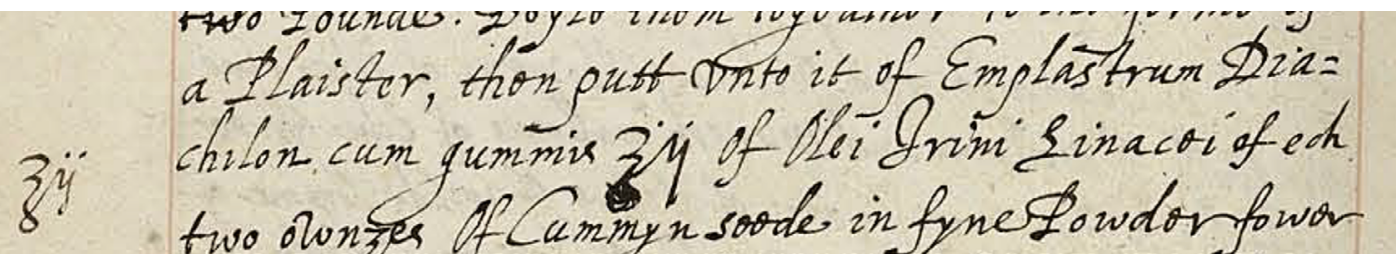

\subsection{Insertion}

The second type of scribal correction found in the manuscript is insertion. When a word or several of them are left out, the scribe resorts to the insertion of the omission, which is made above the line and missing words are thus interpolated in superscript. This is known as interlineation. The exact point of insertion is indicated by means of a caret. This can be seen in the text in figure 10, where the words "or · 3 " have been inserted above the line between "two" and "sponefulles" ("spoonfuls").

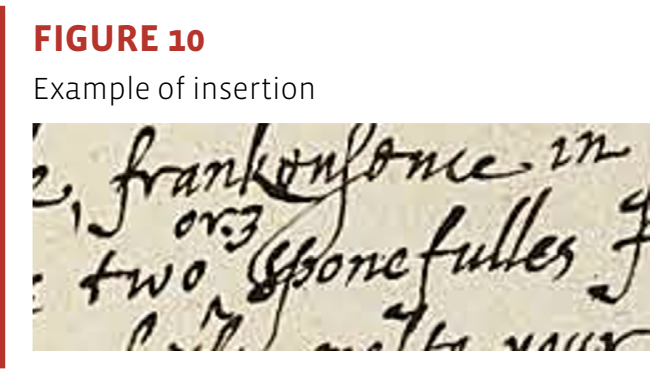

\section{Scribal errors and corrections in our digital edition}

This final section is devoted to explaining how scribal errors and corrections in the manuscript have been included in the digital edition of it, housed at The Malaga Corpus of Early Modern English Scientific Prose. In the "Library" section, the webpage offers free access to all the manuscripts available at the moment, as well as the option to consult the images and text. Once the manuscript has been chosen, a menu at the top is displayed; this provides a list of options including going to the next or previous page, returning to the bookcase, showing 
transcription, etc. If the "Show transcription" option is selected, the transcription will appear to the left in the case of front pages and to the right in that of back pages.

If we pass the cursor over any error in the manuscript image, a dialogue box explaining the type of error or correction will appear (figure 11). We can also check the "Show annotations" option at the top of the page to look for notes. When we click on this, the annotations made to the text are temporarily displayed (figure 12).

\section{FIGURE 11}

Example of treatment of errors and corrections in digital edition (i)

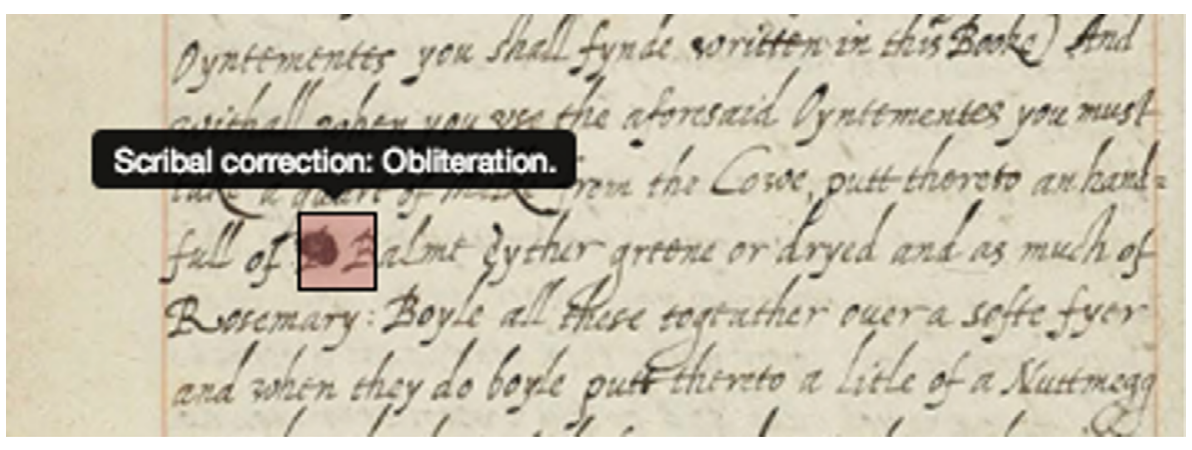

\section{FIGURE 12}

Example of treatment of errors and corrections in digital edition (ii)

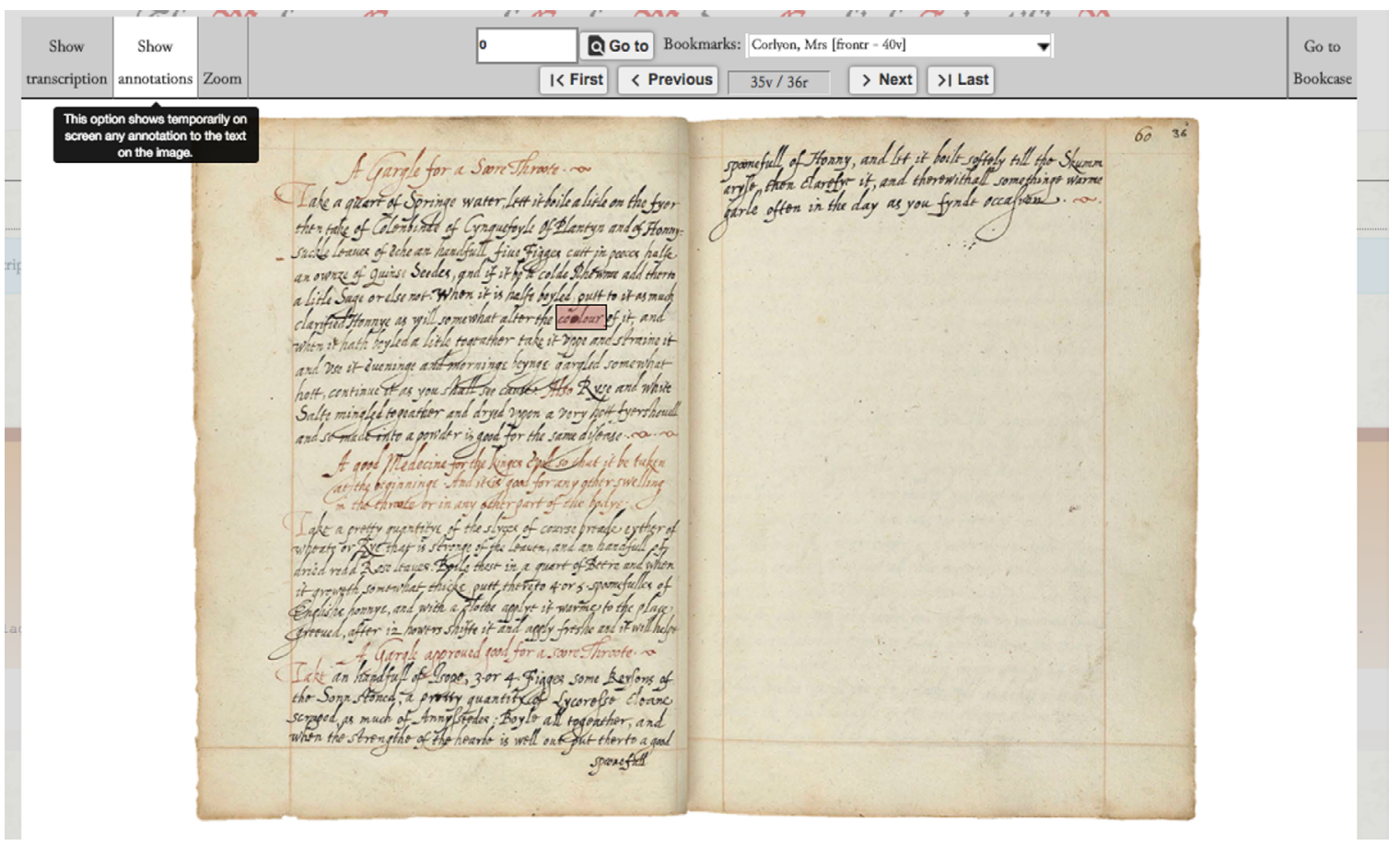


Errors and corrections have also been included in the transcribed text that accompanies the image. As far as insertions are concerned, the word or words inserted have been enclosed within angular brackets and a caret has been added to indicate the point of insertion as it appears in the manuscript.

\section{Final remarks}

The analysis and classification of errors and corrections in manuscripts can be helpful when editing Early English texts, as they can ease the process of revision once the text has been transcribed. Besides, this type of analysis can be useful to determine what the editor should include or not in the edition depending on the aim of it. The process of editing involves making decisions concerning emendation which can be approached from two different perspectives (Moorman, 1975: 56), but between which there is an unlimited number of nuances. In the first one, the scribe is considered an unreliable copyist and therefore the text has to be reconstructed to what the author intended, whereas the second view defends the scribes' authority despite their flaws, since they were closer to the text than any modern scholar can be. It is ultimately the editor who must decide the path to follow.

\section{Works cited}

Calle-Martín, Javier, and others, 2017: The Malaga Corpus of Early Modern English Scientific Prose (MCEMESP), Málaga: University of Málaga [available from http:// modernmss.uma.es/].

CARlquist, Jonas, 2004: "Medieval Manuscripts, Hypertext and Reading. Visions of Digital Editions", Literary and Linguistic Computing 19/1, 105-118.

Leong, Elaine, and Sara PennelL, 2007: "Recipe Collections and the Currency of Medical Knowledge in the Early Modern 'Medical Marketplace'” in Mark S. R. Jenner and Patrick Walus (eds.): Medicine and the Market in England and Its Colonies, c. 1450-c. 1850, New York: Palgrave Macmillan, 133-152.

Moorman, Charles, 1975: Editing the Middle English Manuscript, Jackson: University Press of Mississippi.

Pettı, A. G., 1977: English Literary Hands from Chaucer to Dryden, London: Edward Arnold.

RABE, Jennifer, 2016: "Mediating between Art and Nature: The Countess of Arundel at Tart Hall" in Susanna Burghartz, Lucas Burkart and Christine Göttler (eds.): Sites of Mediation: Connected Histories of Places, Processes, and Objects in Europe and Beyond, 1450-1650, Leiden/Boston: Brill, 183-210. 
TAAVITSAINEN, Irma, and others, 2011: "Medical Texts in 1500-1700 and the Corpus of Early Modern English Medical Texts" in Irma TAavitsaInen and Päivi PaHTA (eds.): Medical Writing in Early Modern English, Cambridge: Cambridge University Press, 9-29. 